$9-2012$

\title{
Controlled Deflection Approach for Rotor Crack Detection
}

Zbigniew Kulesza

Bialystok University of Technology, z.kulesza@pb.edu.pl

Jerzy T. Sawicki

Cleveland State University, j.sawicki@csuohio.edu

Follow this and additional works at: https://engagedscholarship.csuohio.edu/enme_facpub

Part of the Mechanical Engineering Commons

How does access to this work benefit you? Let us know!

\section{Original Citation}

Kulesza, Z., and Sawicki, J.T., 2012, "Controlled Deflection Approach for Rotor Crack Detection," Journal of Engineering for Gas Turbines and Power - Transactions of the ASME, 134(9) pp. 092502.

This Article is brought to you for free and open access by the Mechanical Engineering Department at EngagedScholarship@CSU. It has been accepted for inclusion in Mechanical Engineering Faculty Publications by an authorized administrator of EngagedScholarship@CSU. For more information, please contact library.es@csuohio.edu. 


\author{
Zbigniew Kulesza \\ Faculty of Mechanical Engineering, \\ Bialystok University of Technology, \\ Bialystok, Poland \\ e-mail: z.kulesza@pb.edu.pl \\ Jerzy T. Sawicki \\ Fellow ASME \\ Center for Rotating Machinery \\ Dynamics and Control, (RoMaDyC), \\ Fenn College of Engineering, \\ Cleveland State University, \\ Cleveland, $\mathrm{OH} 44115-2214$ \\ e-mail: j.sawicki@csuohio.edu
}

\section{Controlled Deflection Approach for Rotor Crack Detection}

A transverse shaft crack is a serious malfunction that can occur due to cyclic loading, creep, stress corrosion, and other mechanisms to which rotating machines are subjected. Though studied for many years, the problems of early crack detection and warning are still in the limelight of many researchers. This is due to the fact that the crack has subtle influence on the dynamic response of the machine and still there are no widely accepted, reliable methods of its early detection. This paper presents a new approach to these problems. The method utilizes the coupling mechanism between the bending and torsional vibrations of the cracked, nonrotating shaft. By applying an external lateral force of constant amplitude, a small shaft deflection is induced. Simultaneously, a harmonic torque is applied to the shaft inducing its torsional vibrations. By changing the angular position of the lateral force application, the position of the deflection also changes opening or closing of the crack. This changes the way the bending and torsional vibrations are being coupled. By studying the coupled lateral vibration response for each angular position of the lateral force one can assess the possible presence of the crack. The approach is demonstrated with a numerical model of a rotor. The model is based on the rigid finite element method (RFE), which has previously been successfully applied for the dynamic analysis of many complicated, mechanical structures. The RFE method is extended and adopted for the modeling of the cracked shafts. An original concept of crack modeling utilizing the RFE method is presented. The crack is modeled as a set of spring-damping elements (SDEs) of variable stiffness connecting two sections of the shaft. By calculating the axial deformations of the SDEs, the opening/closing mechanism of the crack is introduced. The results of numerical analysis demonstrate the potential of the suggested approach for effective shaft crack detection.

\section{Introduction}

One of the most dangerous malfunctions of rotating machines are shaft cracks. Transverse cracks occur due to cyclic loading, thermal stresses, creep, corrosion, and other mechanisms to which rotating shafts are subjected. Once a crack has appeared, high stresses develop at its tip and allow the crack to propagate deeper, even if external loads are not changing. When the crack has propagated to a relevant depth, the propagation speed increases dramatically and the shaft may fail in a very short time, what usually leads to a catastrophic accident. That is why an early detection of the potential shaft cracks inside the rotating machine components is so important.

There are numerous published contributions on the subject of crack modeling, early detection, location and estimation of severity of cracks. Usual crack detection methods are based on vibration signal analysis [1-3]. By studying the changes in the vibration spectra, the appearance of the possible shaft crack can be confirmed (or declined). The frequently discussed changes in frequency spectra induced by a crack are: a considerable increase of the amplitude of the synchronous frequency $1 \mathrm{X}$ and an appearance of its second multiple $2 X$ [3]. However, such symptoms are characteristic not only for cracked rotors, but can be induced by other faults such as: bearing malfunctions, misalignment, thermal sensitivity, etc. [1].

Other methods include changes in rotor modal parameters such as its natural frequencies and mode shapes [3,4], state observers [5,6], Kalman filters [7], genetic algorithms [8], wavelet $[9,10]$ or Huang-Hilbert transforms [11], and many others.

A relatively new approach to the problem of rotor crack detection employs the use of a specially designed diagnostic force applied to the rotating shaft [12-15]. If the force is harmonic, then
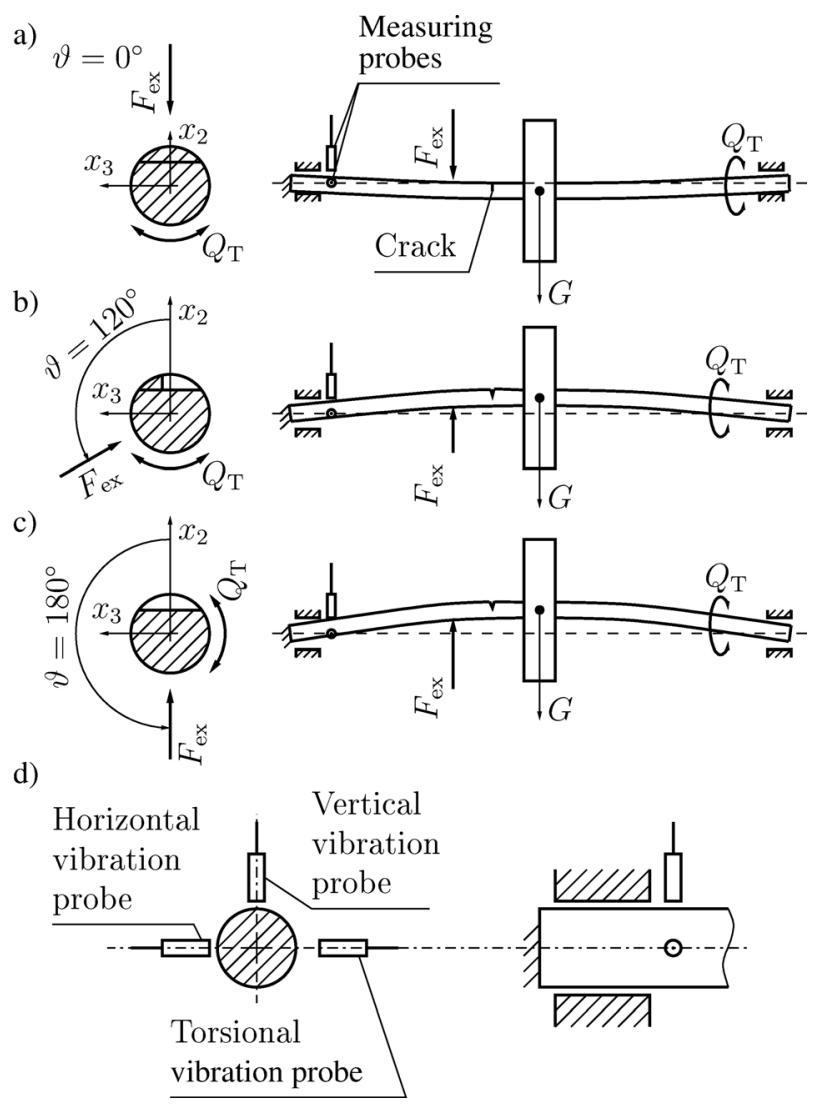

Fig. 1 Schematic diagrams of the method for different angular positions of the external force: (a) $\vartheta=\theta$ deg, fully closed crack; (b) $\vartheta=\mathbf{1 2 0} \mathrm{deg}$, partially open crack; (c) $\vartheta=\mathbf{1 8 0} \mathrm{deg}$, fully open crack; and $(d)$ arrangement of the measuring probes 
a)
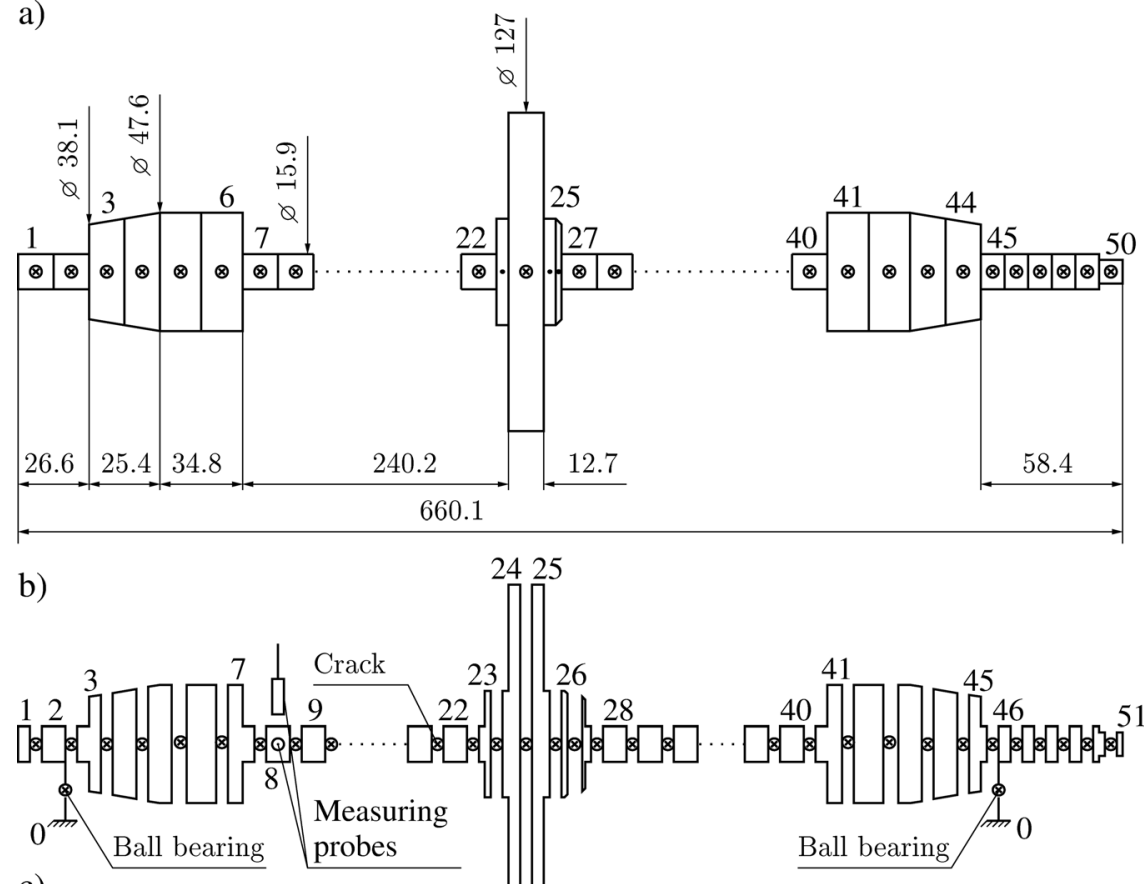

c)

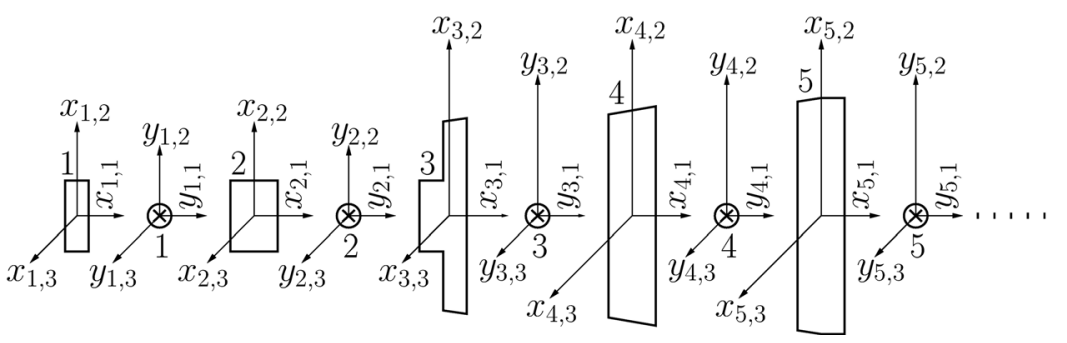

Fig. 2 Rigid finite element model of the rotor: (a) original division into 50 springdamping elements, $(b)$ secondary division into 51 rigid finite elements, and $(c)$ local coordinate systems of RFEs $\left(x_{r, 1}, x_{r, 2}, x_{r, 3}\right)$ and $\operatorname{SDEs}\left(y_{k, 1}, y_{k, 2}, y_{k, 3}\right)$

the presence of the crack generates responses containing frequencies at combinations of the angular speed, applied forcing frequency, and the rotor natural frequencies [14]. It has been shown that the appearance of the combinational frequencies is a very strong signature of the shaft crack $[14,15]$. However, the research conducted so far has focused on applying the harmonic force, acting in one fixed direction only [13-15].

A well known feature of the cracked shaft is the coupling between the lateral and torsional vibrations. The appearance of coupled bending and torsional vibrations can be utilized as a possible shaft crack indicator, which has been reported by several authors [16,17].

Similar to the previous methods, the present paper recommends the use of the additional diagnostic force applied perpendicularly to the shaft axis. However, the amplitude of this force remains constant, while the angular location of its application (i.e., line of action) changes from $0 \mathrm{deg}$ to $360 \mathrm{deg}$. Moreover, the shaft is not rotating but excited by an additional torque inducing its torsional vibrations. The proposed method is based on vibration signal analysis, namely on the coupling mechanism between the lateral and torsional vibrations.

The approach is demonstrated by the numerical model of the rotor. The model utilizes the rigid finite element (RFE) method, which was formulated by Kruszewski et al. [18]. Rigid finite element method is based on a completely different approach than the classical finite element method (FEM).

A given mechanical structure (such as a bar, beam, frame, or shell) is discretized into a finite number of rigid elements, containing inertial features of the structure. These rigid elements are con- nected by massless and nondimensional spring-damping elements (SDEs), representing the stiffness and damping of the structure. The method has been successfully applied for the dynamic analysis of mechanisms, machine tools, cranes, ship drive systems, and even ship hulls [18]. Lately the method has been improved and extended by Wittbrodt et al. [19], who used it for successful dynamic calculations of flexible multibody systems with changing configuration such as robot manipulators.

The idea of applying the rigid finite element method for modeling driving shafts is not new [18]. However, it is the first time that RFEs will be used for modeling the opening/closing of the shaft crack.

\section{Concept of the New Method for Rotor Crack Detection}

A schematic diagram explaining the concept of the proposed method is shown in Fig. 1. The nonrotating cracked rotor is subjected to a harmonic torsional excitation $Q_{\mathrm{T}}$ and simultaneously to the constant external force $F_{e x}$ applied perpendicularly to the bearings centerline.

The force is applied at different angles $\vartheta$, causing some small deflections of the shaft. By changing the angular position of the force, the position of the resulting deflection also affects opening or closing of the crack. This changes the stiffness of the shaft and the way the bending and torsional vibrations are being coupled. It is supposed that by studying the coupled bending vibration response for each angular position of the external force one will be able to assess the possible presence of the crack. 

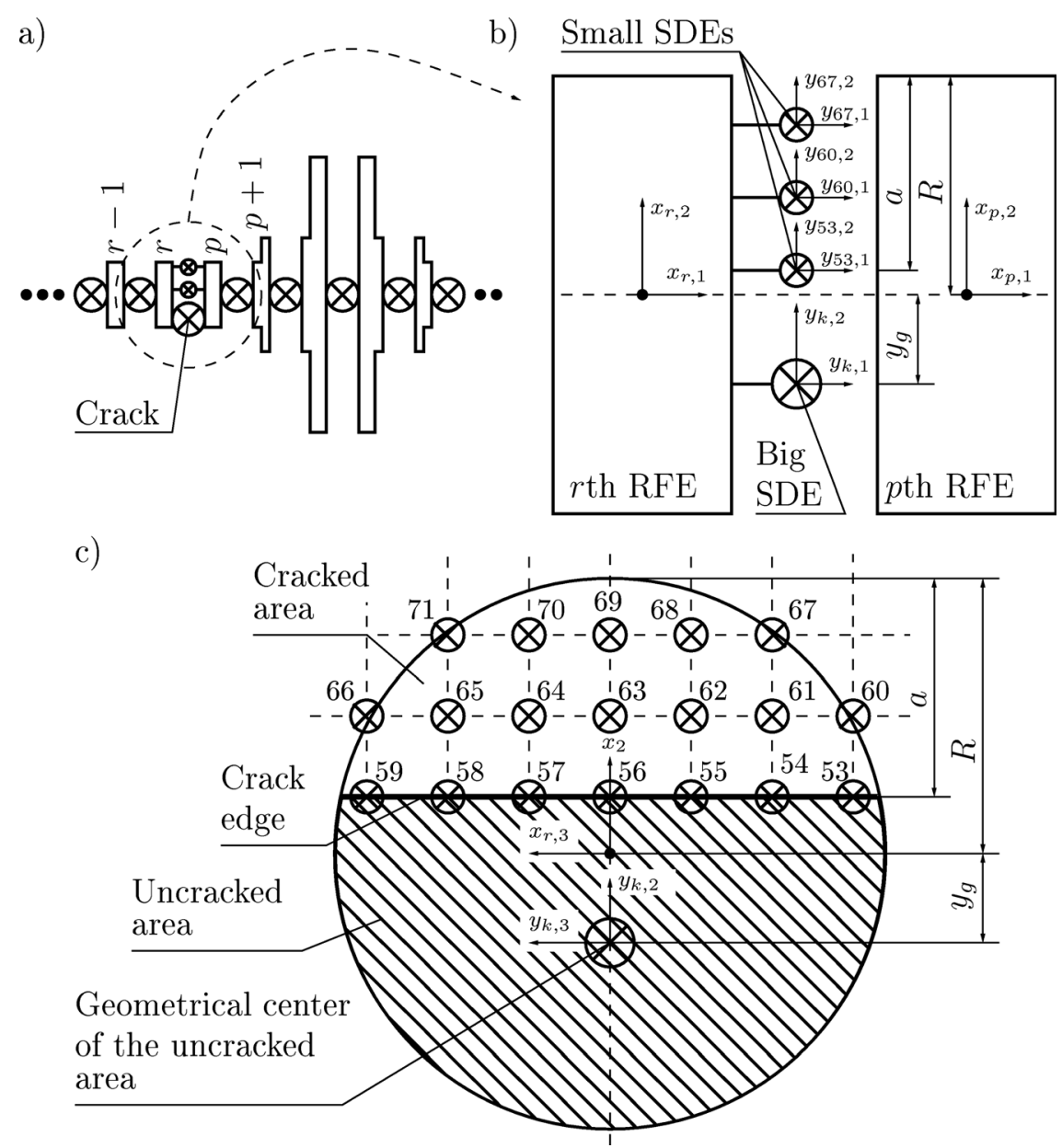

Fig. 3 Model of the crack: (a) possible location of the crack, (b) two RFEs and several SDEs at the location of the crack, and (c) shaft cross section at the location of the crack
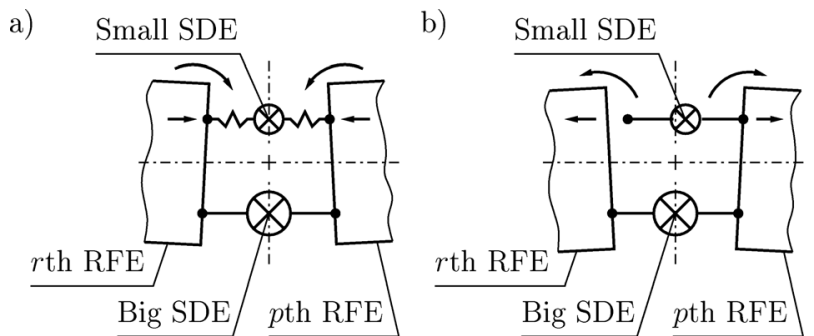

Fig. 4 Possible deformations of the small SDE: (a) compression and $(b)$ tension

\section{Rigid Finite Element Model of the Rotor}

The proposed approach will be demonstrated on a rotor supported by two ball bearings, consisting of a shaft and a disk (Fig. 2). The rotor is a part of a crack detection test rig utilized at the Center for Rotating Machinery Dynamics and Control (RoMaDyC) at Cleveland State University [14]. Main dimensions and the schematic diagram of the rotor are presented in Fig. 2(a).

Using the rigid finite element method $[18,19]$, the rotor is divided into a selected number of lumped elements. The division runs in two steps.

In the first step, the rotor is divided into 50 elements (Fig. 2(a)). In the middle of each prismatic element, a spring-damping element is located. In Fig. 2(a) SDEs are marked with crossed

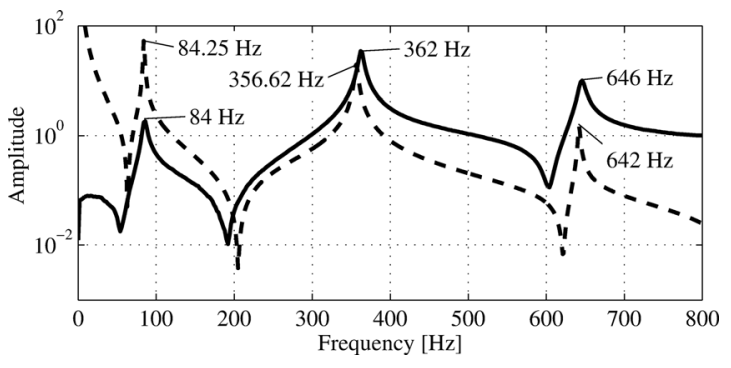

Fig. 5 Frequency transfer function of the free-free rotor (continuous line) and its RFE model (dashed line)

circles. Each of tapered elements, numbered as 3, 4, and 43, 44 is replaced by six prismatic elements of equal length and gradually decreasing diameter, according to the procedure described in $[18,19]$. The motion of each SDE is considered in an independent coordinate system with its three main axes $y_{k, 1}, y_{k, 2}$, and $y_{k, 3}$ (Fig. $2(c)$ ). The main axes of each SDE are chosen in such a way that the force acting along each axis causes only a translational deformation in a direction along the axis. Similarly, the pair of forces acting around each coordinate axis causes only a torsional deformation around the axis.

In the second step, 51 rigid finite elements are located between the corresponding SDEs obtained in the first step. As in the first step, nonprismatic elements (e.g., 3, 4, 5) are replaced by six prismatic RFEs, according to [18,19]. 

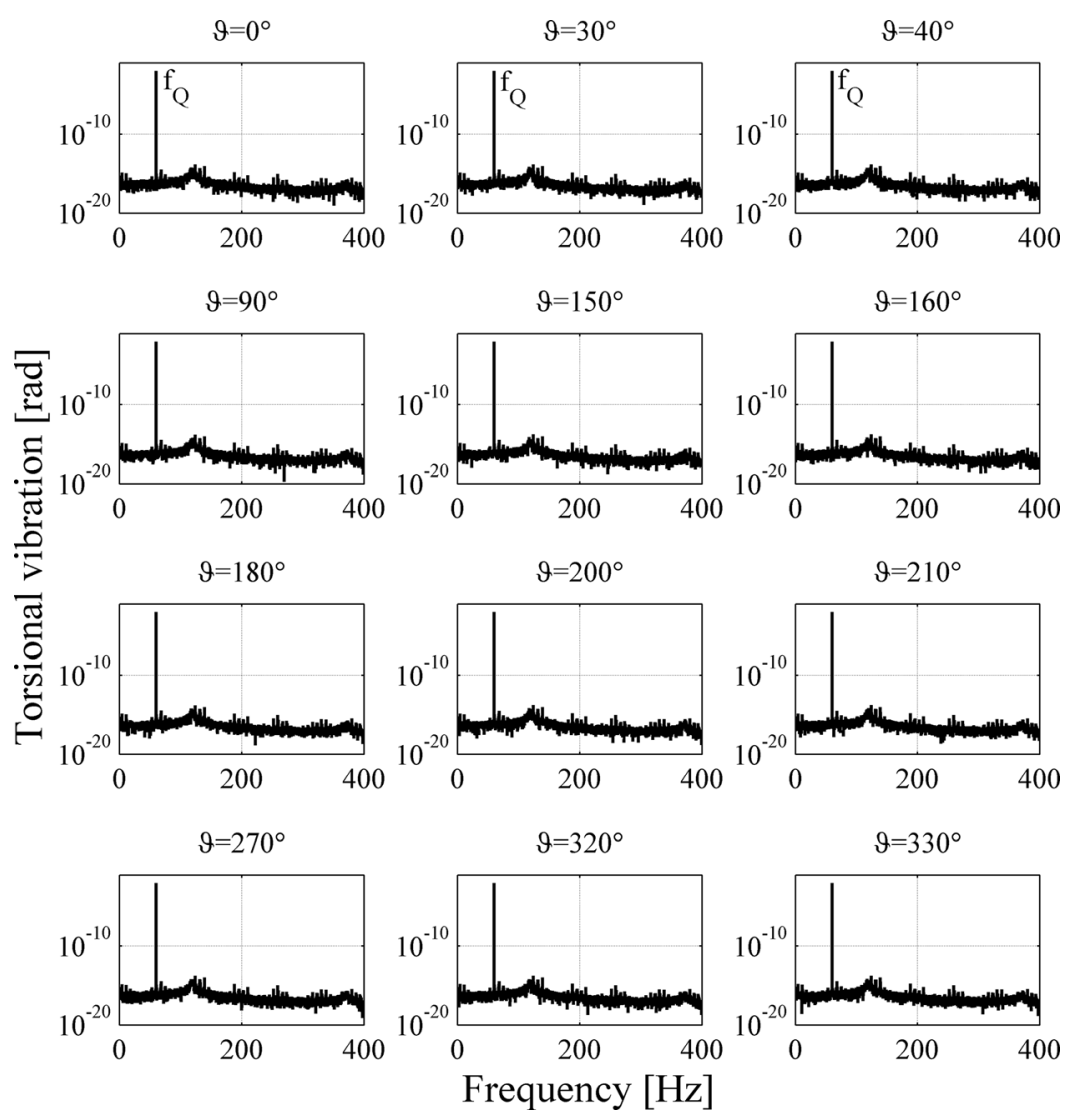

Fig. 6 Torsional response for different angles $\vartheta$; uncracked shaft; $f_{Q}=60 \mathrm{~Hz}$
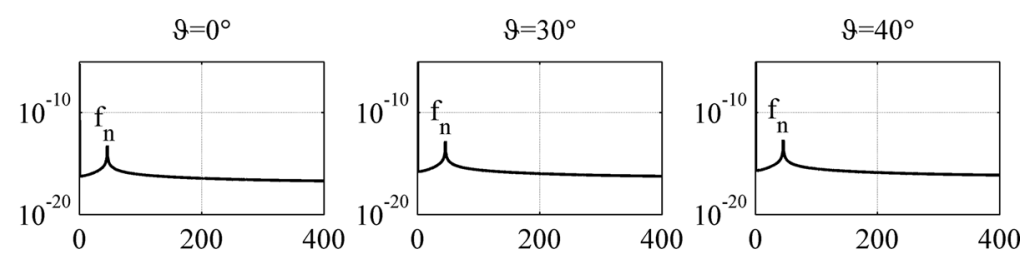

$\vartheta=90^{\circ}$
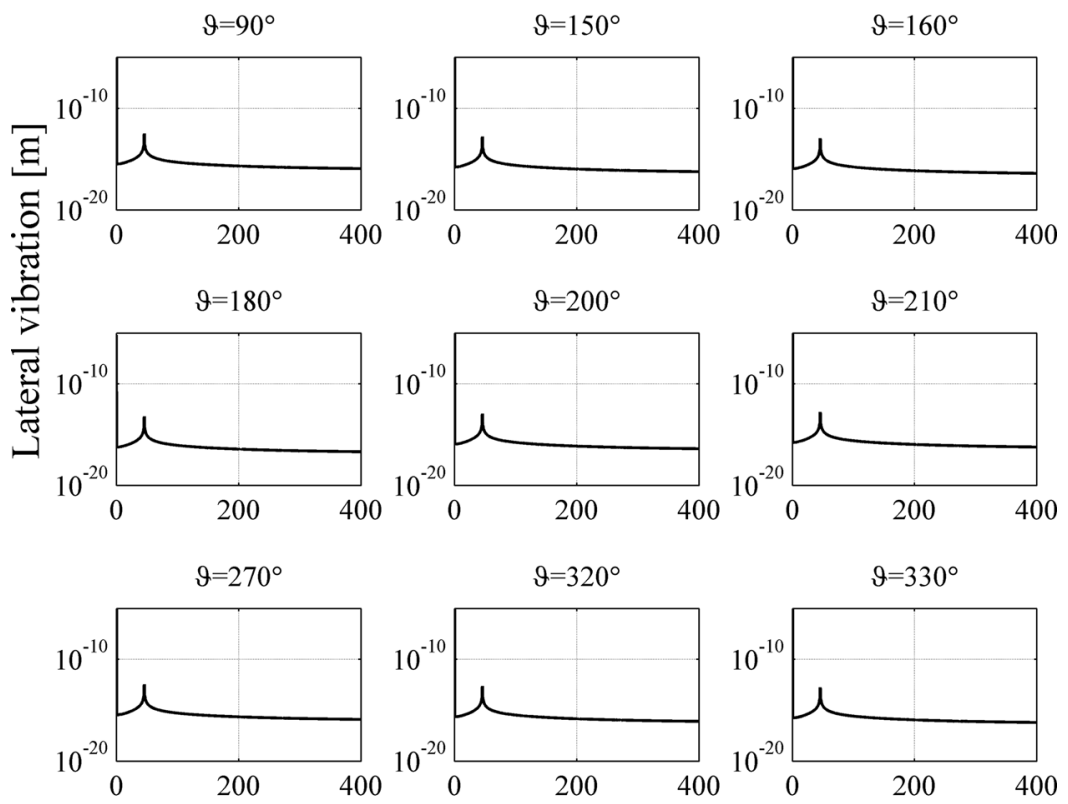

Frequency [Hz]

Fig. 7 Bending response for different angles $\vartheta$; uncracked shaft; $f_{Q}=60 \mathrm{~Hz}$ 

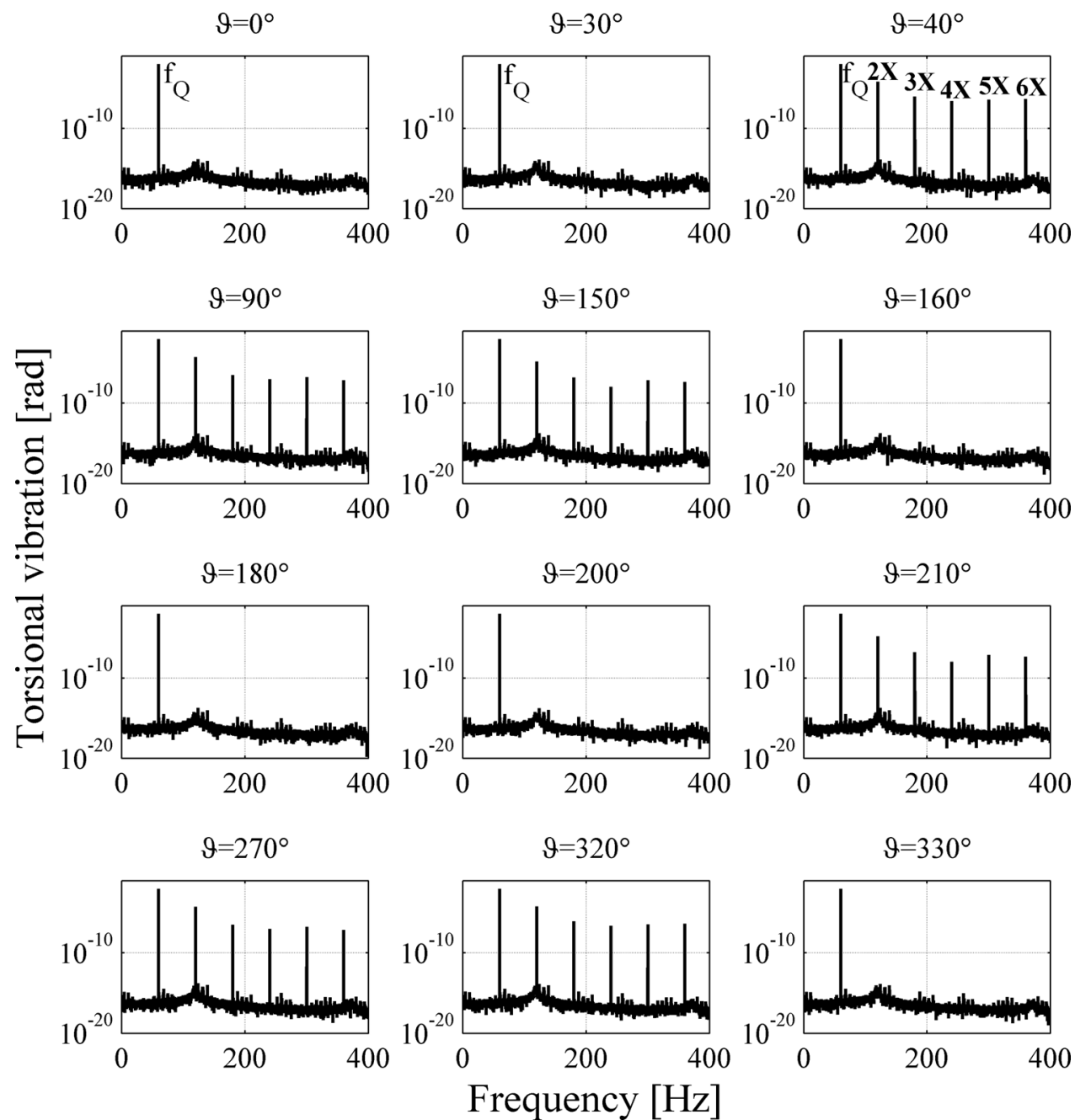

Fig. 8 Torsional response for different angles $\vartheta ; 25 \%$ crack; $f_{Q}=60 \mathrm{~Hz}$

The motion of each RFE is considered in an independent coordinate system with three main axes $x_{r, 1}, x_{r, 2}, x_{r, 3}$ (Fig. 2(c)). The center of the system is located at the mass center of a given element. At a standstill, axes $x_{r, 1}, x_{r .2}$, and $x_{r .3}$ coincide with three principal axes of the element; $x_{r, 1}$ coincides with the rotation axis.

The bearings are modeled using two additional spring-damping elements (numbered as 50 and 51) connecting the basis (numbered as 0) with the 2nd and with the 46th RFE. The crack is located between the 21 st and the 22nd RFE, while the probes measuring the lateral and torsional vibrations of the rotor are near the center of the 8th RFE.

Rigid finite elements are characterized by their masses and mass moments of inertia, while spring-damping elements by their stiffness and damping coefficients. The masses of spring-damping elements are neglected. Static characteristic of each springdamping element is linear, which means that force loading the element is the sum of two forces: the one that is proportional to deformation (stiffness) and the other that is proportional to deformation velocity (damping). Spring-damping elements are loaded with translational forces, as well as with torques.

After dividing the rotor into spring-damping elements and after assuming their coordinate systems, the flexural and torsional stiffness coefficients along each six directions of each element are calculated according to the procedure described in $[18,19]$. Similarly, masses and mass moments of inertia of all 51 RFEs are determined $[18,19]$. Then, on the basis of stiffness coefficients, masses and mass moments of inertia of individual SDEs and RFEs, stiffness $\mathbf{K}$, and mass $\mathbf{M}$ matrices of the rotor are created [18,19].
Proportional damping is assumed with the damping matrix D calculated according to the known formulas $[18,19]$

$$
\mathbf{D}=\star_{D} \mathbf{M}+\beta_{D} \mathbf{K}
$$

where coefficients $\alpha_{D}$ and $\beta_{D}$ are assumed as follows: $\alpha_{D}=10^{-5}$, $\beta_{D}=\theta$.

The equation of motion of the rotor takes the following form $[18,19]$ :

$$
\mathbf{M} \ddot{\mathbf{q}}+\mathbf{D} \dot{\mathbf{q}}+\mathbf{K q}=\dot{\mathbf{G}}+\mathbf{F}_{e x}+\mathbf{Q}_{\mathrm{T}}
$$

where $\mathbf{q}$ is a vector of general displacements of the centers of masses of individual RFEs, $\mathbf{G}, \mathbf{F}_{e x}, \mathbf{Q}_{\mathrm{T}}$ are vectors of the following forces: gravity, external force perpendicular to the shaft axis, and external torque exciting one end of the shaft (Fig. 1).

\section{Model of the Crack}

The crack is usually modeled by periodical stiffness changes, resulting from the so called "breathing" mechanism, i.e., from the periodical opening and closing of the crack due to the rotational motion of the rotor. The very simple "hinge models" were the first, which included the breathing of the crack by introducing two different states: the completely open and the completely closed crack at particular angular positions of the rotor [20,21]. Mayes and Davies [22] introduced the model in which the changes from 


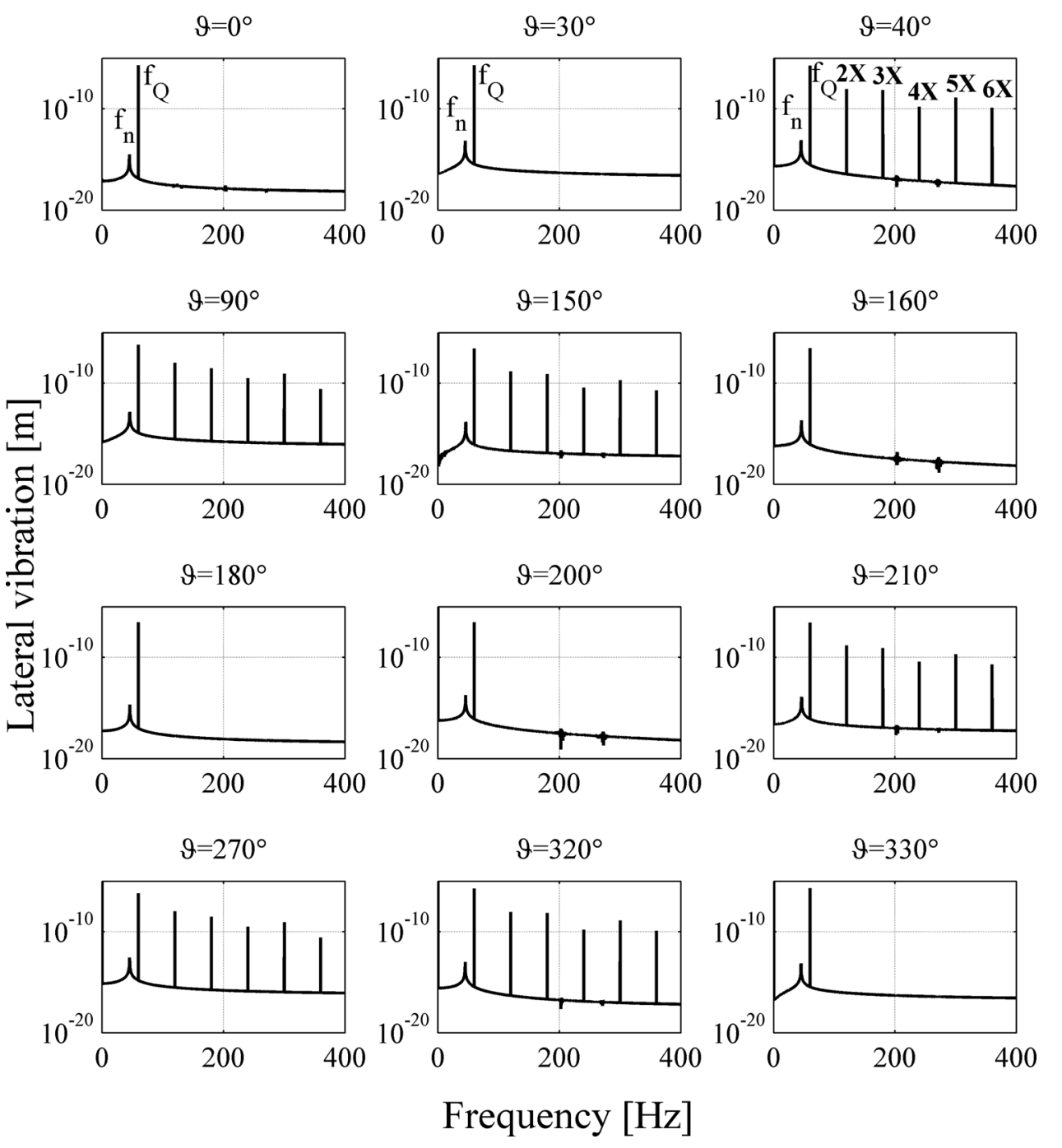

Fig. 9 Bending response for different angles $\vartheta ; 25 \%$ crack; $f_{Q}=60 \mathrm{~Hz}$

the completely open to the completely closed state were described by a continuous function (the so called "crack steering function"). Usually this function has the form of a simple cosine function depending on the torsional angle.

Progressive development of the finite element method and its application to rotor dynamics $[23,24]$ resulted in very sophisticated crack models in which fracture mechanics methods are applied. Dimarogonas and Paipetis [25] introduced the full, $6 \times 6$ stiffness matrix for the finite element containing the continuously open crack (the so called "slotted crack"). Darpe et al. [17] presented the detailed derivation of the full stiffness matrix of the cracked finite element including an original model of the crack breathing. Using their approach, the extent of crack opening/closing is evaluated by calculating the stresses at selected points along the crack edge line. The positive sign of these stresses corresponds to the compressive state of the given portion of the cracked surface, and the closed crack, while the negative sign corresponds to the tensile state and the open crack.

All these crack models have been developed for the rotating shafts. However, if there is no rotational motion, then the models based on the crack steering function are not very suitable. This is due to the fact that they depend on the torsional angle. That is why, in case of nonrotating shafts, the models should be used where stresses (or deformations) at the crack location are calculated $[17,24]$.
The present article introduces a new model of the crack. As the whole rotor is modeled using the rigid finite element method, the crack is modeled by several spring-damping elements. Similar to [17], the crack opening/closing is evaluated by the very small deformations of the SDEs at the location of the crack. The details about the suggested model are explained below.

The crack is supposed to be located between the two RFEs and is modeled using several spring-damping elements connecting these RFEs. Figure 3 shows two rigid finite elements and the cross section of the shaft at the location of the crack. The crack edge line located at depth $a$ from the side surface of the shaft separates the uncracked (hatched) and cracked areas.

The main SDE connecting the two RFEs (marked with a big crossed circle in Fig. 3(b)) is located at the geometrical center of the uncracked area. Stiffness coefficients $k_{C, j}$ of this SDE in the main six directions are constant and proportional to the relative depth of the uncracked area

$$
k_{C, j}=(1-\mu) k_{U, j}
$$

where $\mu=a / 2 R$ is the relative depth of the crack, $R$ is the radius of the shaft, $k_{U, j}$ are stiffness coefficients of the SDE, which would connect the two RFEs in case of the uncracked shaft, $j=1,2, \ldots, 6$. Stiffness coefficients $k_{U, j}$ are calculated using the procedure described in $[18,19]$. 
$\vartheta=0^{\circ}$

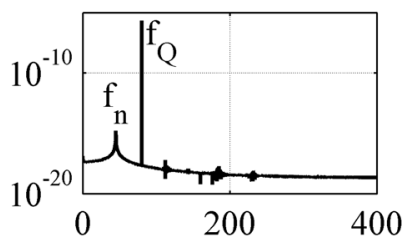

$\vartheta=90^{\circ}$

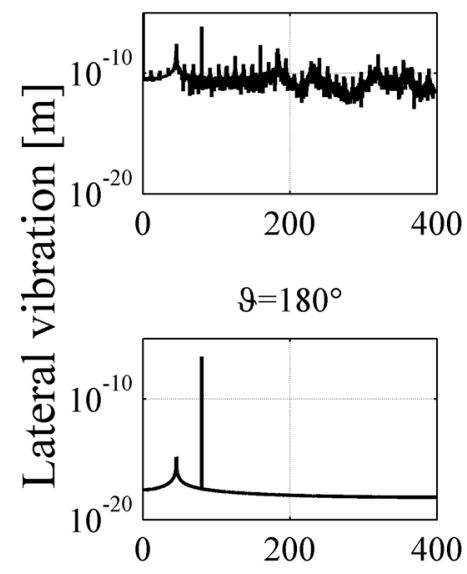

$\vartheta=270^{\circ}$

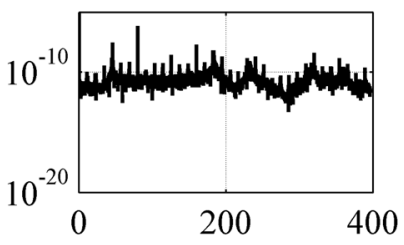

$\vartheta=30^{\circ}$

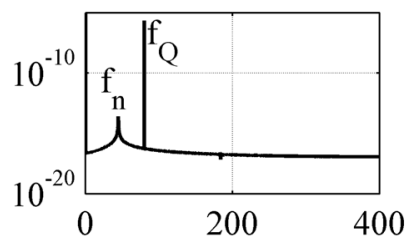

$\vartheta=150^{\circ}$

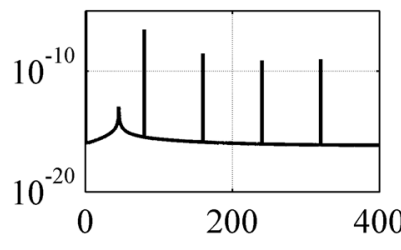

400
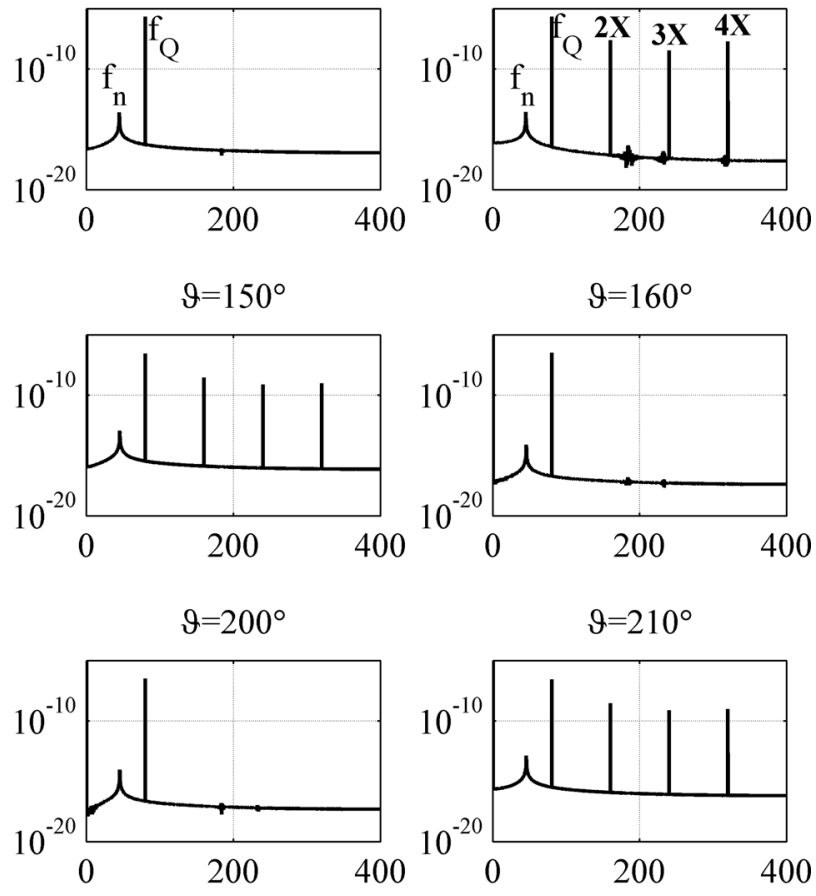

$\vartheta=160^{\circ}$

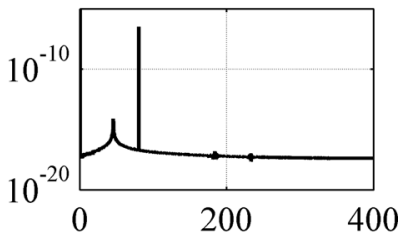

$\vartheta=320^{\circ}$

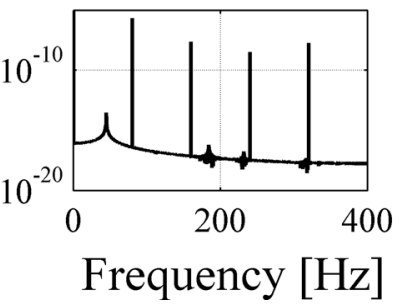

$\vartheta=330^{\circ}$

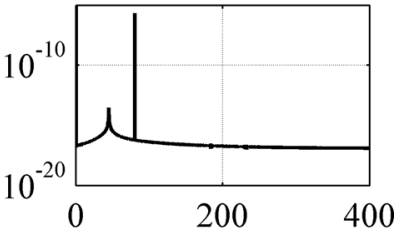

Fig. 10 Bending response for different angles $\vartheta ; 25 \%$ crack; $f_{Q}=80 \mathrm{~Hz}$

The cracked area between the two RFEs is connected with several smaller SDEs. There are 19 SDEs (numbered from 53 to 71) in Fig. 3(c). In practical calculations the number of small SDEs should be chosen in a way ensuring the realistic opening and closing of the crack. These SDEs are located at selected points of the uncracked area, e.g., at the nodes of the imaginary geometrical array, formed with vertical and horizontal lines running perpendicular and parallel to the crack edge. Stiffness coefficients $k_{k, j}$ of the individual SDEs are equal and are chosen in such a way that their sums in all six directions are proportional to the relative depth of the crack

$$
\sum_{k=53}^{53+n_{C}} k_{k, j}=\mu k_{U, j}
$$

where $n_{C}$ is the number of small SDEs. Hence the sum of the stiffness coefficients of small SDEs $k_{k, j}$ and a big SDE $k_{C, j}$ in the $j$ th direction equals the stiffness of the uncracked SDE $k_{U, j}$,

$$
k_{C, j}+\sum_{k=53}^{53+n_{C}} k_{k, j}=k_{U, j}
$$

Crack closing and opening is included by stiffness changes of small SDEs. It is assumed that the stiffness of an individual small $\mathrm{SDE}$ in a given direction $j$ can be zero or $k_{k, j}$ depending on the deformation $\Delta w_{k, 1}$ of this SDE along axis $y_{k, 1}$ (for $j=4$, i.e., along the axis of the shaft). If this deformation is greater than zero, then the corresponding SDE is compressed, its stiffness should be $k_{k, j}$ and the crack at its location is closed. It can be vividly presented, as if the spring damper touches the crack surface (Fig. 4). On the other hand, if this deformation is less than or equal to zero, then the corresponding SDE is under tension, its stiffness should be zero and the crack at its location is opened. It can be presented as if the spring damper does not touch the surface of the crack. This way, based on the sign of the deformation of all small SDEs in a direction perpendicular to the crack surface, the open/close state of the crack can be easily determined, and the corresponding stiffness change can be introduced into the stiffness matrices.

Deformations of a given SDE are expressed by vector $\Delta \mathbf{w}_{k}$ composed of six components: the first three are translational deformations along corresponding axes $y_{k, 1}, y_{k, 2}, y_{k, 3}$, and the next three are rotational deformations around the same axes. The first component $\Delta w_{k, 1}$ is used for determining the opening or closing of the crack, as it is described above. According to $[18,19]$, vector $\Delta \mathbf{w}_{k}$ is calculated as follows:

$$
\Delta \mathbf{w}_{k}=\mathbf{T}_{r, k} \mathbf{q}_{r}-\mathbf{T}_{p, k} \mathbf{q}_{p}
$$

where $\mathbf{q}_{r}$ and $\mathbf{q}_{p}$ are vectors of displacements of the $r$ th and $p$ th RFE connected with the $k$ th SDE, and $\mathbf{T}_{r, k}$ and $\mathbf{T}_{p, k}$ are corresponding transformation matrices $[18,19]$.

Hence the procedure of determining the crack open/close state is performed according to the following steps: 

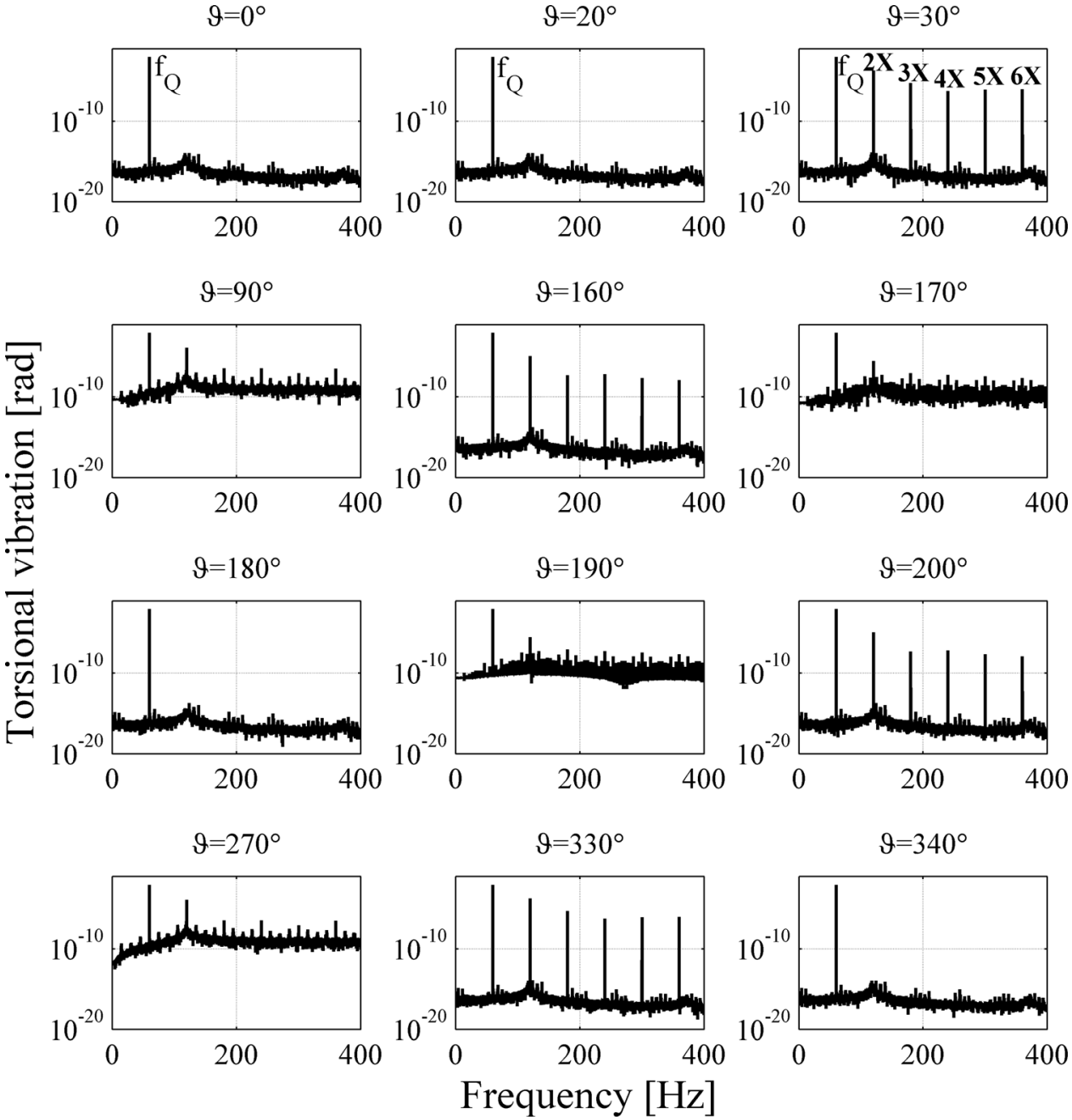

Fig. 11 Torsional response for different angles $\vartheta ; 40 \%$ crack; $f_{Q}=60 \mathrm{~Hz}$

1. Initially the crack is supposed to be completely opened, i.e., stiffness matrices of all small SDEs are zero and only the stiffness matrix of the big SDE is introduced into the stiffness matrix $\mathbf{K}$ of the whole system (Eq. (2)).

2. For the given time step, the response vector $\mathbf{q}$ is calculated according to Eq. (2) and vectors of displacements $\mathbf{q}_{r}$ and $\mathbf{q}_{p}$ of the two RFEs between the crack are excluded from it.

3. Deformation vectors $\Delta \mathbf{w}_{k}$ of all small SDEs are calculated, according to Eq. (6).

4. If the first component $\Delta w_{k, 1}$ of the given vector $\Delta \mathbf{w}_{k}$ is less than or equal to zero, then the corresponding SDE is open and its stiffness matrix remains zero. Otherwise, the corresponding SDE is closed and its stiffness matrix is modified to contain $k_{k, j}$ components.

5. Stiffness matrix $\mathbf{K}$ of the whole system is updated with modified matrices of all small SDEs and the procedure from 2 to 5 is repeated with the new value of $\mathbf{K}$.

\section{Results}

To evaluate the quality of the proposed RFE approach the values of the frequency transfer function have been calculated and compared with the values obtained experimentally. Figure 5 presents such a comparison for the uncracked, free-free rotor (with no supporting bearings) obtained experimentally using the impact hammer modal testing (continuous line) and from the RFE model (dashed line). During the experiment the rotor suspended on a light thin string was impacted near the center of the 17th RFE and its vibrations were measured at the center of the 15th RFE. Very good agreement between the experimental and numerical data can be observed; the values of the first three natural frequencies, which for the experiment are $84.25,362$, and $646 \mathrm{~Hz}$, agree very well with the values obtained from the RFE model $(84,356.62$, and $642 \mathrm{~Hz}$, respectively). Natural frequencies of the uncracked rotor supported by ball bearings are different than those of the free-free rotor. In this case the first two bending frequencies are located at $f_{n}=45 \mathrm{~Hz}$ and $f_{n}=225$ $\mathrm{Hz}$, while the first torsional frequency is at $f_{T}=423 \mathrm{~Hz}$. This is due to additional stiffness and damping introduced by bearings.

During the numerical analysis three different models of the rotor have been considered: the first with no crack, the second with a $25 \%$ deep crack, and the third with a $40 \%$ deep crack. The number of small spring-damping elements connecting the $21 \mathrm{st}$ and the 22 nd elements of the shaft was $n_{C}=260$ for the $25 \%$ deep crack and $n_{C}=\$ 488$ for the $40 \%$ deep crack. In all cases the value of the lateral force was $F_{e x}=40 \mathrm{~N}$, while the form of the external torque $Q_{T}=A_{Q} \sin \left(2 \pi f_{Q} t\right)$, where the amplitude $A_{Q}=50$ $\mathrm{Nm}$. The torque can be also presented as $Q_{T}=A_{Q} \sin \left(2 \pi n_{Q}+\phi_{Q}\right)$, where $\phi_{Q}$ and $n_{Q}$ are its phase and number of cycles, respectively $\left(0 \leq \phi_{Q} \leq 2 \pi\right)$. The force and the torque were applied at the center of the 35th and 49th RFE for the force and torque, respectively (Fig. 2(b)). Two different frequencies of the exciting torque have been considered: $f_{Q}=\$ 60 \mathrm{~Hz}$ and $f_{Q}=\$ 80 \mathrm{~Hz}$. 

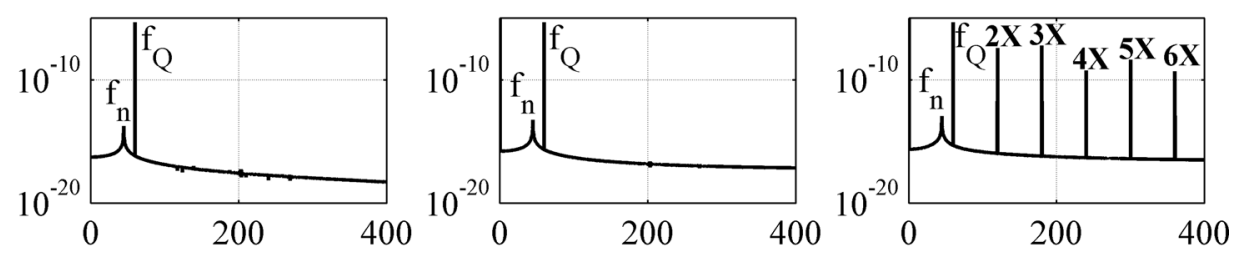

$\vartheta=90^{\circ}$
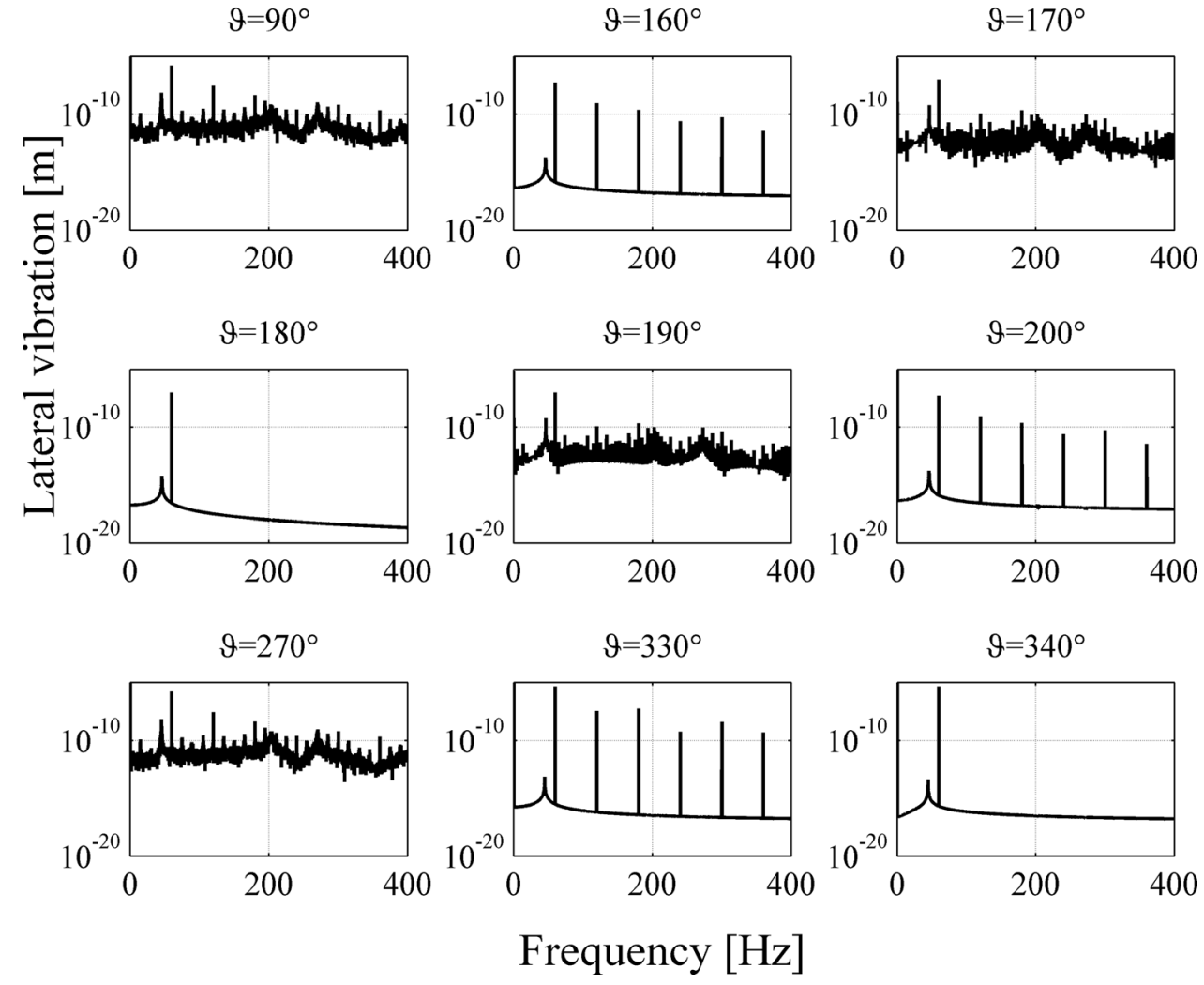

\section{Frequency $[\mathrm{Hz}]$}

Fig. 12 Bending response for different angles $\vartheta ; 40 \%$ crack; $f_{Q}=60 \mathrm{~Hz}$

The calculations have been conducted for the rotor made of steel of Young's modulus $E=2.08 \times 40^{11} \mathrm{~Pa}$, Poisson's ratio $\nu \psi=0.3$, and density $\rho=7850 \mathrm{~kg} / \mathrm{m}^{3}$. The values of the radial bearings' stiffness and damping were assumed as $k_{B}=3.6 \times 40^{6}$ $\mathrm{N} / \mathrm{m}$, and $d_{B}=10 \mathrm{~N} \mathrm{~s} / \mathrm{m}$. Furthermore, the torsional stiffness and damping at the left bearing were chosen to be $k_{T}=44 \times 40^{4} \mathrm{~N} \mathrm{~m} /$ $\mathrm{rad}$ and $d_{T}=20 \mathrm{Nms} / \mathrm{rad}$, as the left end of the shaft is fixed (Fig. 1).

Figures 6-13 present frequency responses for different angles $\vartheta \psi$ of the lateral force $F_{e x}$. Bending response is shown only for the horizontal $x_{3}$ axis, as the vibrations along axes $x_{2}$ and $x_{3}$ are much the same.

Figures 6 and 7 present torsional and bending responses of the uncracked rotor. As expected, the torsional spectrum contains only one component of the exciting torque frequency $f_{Q}=\$ 60 \mathrm{~Hz}$. In the bending response only the first natural frequency $f_{n}=\nless 45$ $\mathrm{Hz}$ is slightly induced. Such characteristics are typical for the linear model of the rotor.

Figures 8, 9, and 10 present responses of the $25 \%$ cracked rotor. Due to the nonlinearities introduced by the crack, subsequent integer multiples of the exciting torque frequency $f_{Q}$ denoted as $2 \mathrm{X}$ $(120 \mathrm{~Hz}), 3 \mathrm{X}(180 \mathrm{~Hz}), 4 \mathrm{X}(240 \mathrm{~Hz})$, etc. appear in the torsional response (Fig. 8). However, all these frequencies are observed only for particular angles $\vartheta$, i.e., for $\vartheta$ from $40 \mathrm{deg}$ to $150 \mathrm{deg}$ and for $\vartheta$ /from $210 \mathrm{deg}$ to $320 \mathrm{deg}$. It should be noticed that such angle ranges correspond to the situations when the crack is partially open. For other ranges, only one component is present in the vibration spectra. This is the frequency of the exciting torque $f_{Q}$. In this case, the angles are near 0 deg and $180 \mathrm{deg}$, what corresponds to the (almost) fully open and (almost) fully closed crack.

The similar, yet more important situation, is in the bending spectra (Figs. 9 and 10), where for the same angle ranges the same frequency components can be observed (including the multiples $2 \mathrm{X}, 3 \mathrm{X}$, and so on). For other angle ranges, the bending frequency spectrum contains only slightly induced natural frequency $f_{n}$ and exciting torque frequency $f_{Q}$.

The rotor with a $40 \%$ deep crack excited with a $60 \mathrm{~Hz}$ torque behaves similarly (Figs. 11 and 12), yet the angle ranges for which additional bending frequencies are induced are wider: from $\vartheta \psi=30 \mathrm{deg}$ to $\vartheta \psi=160 \mathrm{deg}$ and from $\vartheta \psi 200 \mathrm{deg}$ to $\vartheta \vartheta=330 \mathrm{deg}$. This would suggest that for deeper cracks it is more difficult to completely close (or completely open) the crack and consequently not to induce the additional bending frequencies.

The situation for a $40 \%$ cracked rotor excited with a $80 \mathrm{~Hz}$ torque is completely different (Fig. 13). For most angular positions of the lateral force the bending response contains a full spectrum of different frequencies of relatively high amplitudes. Only for $\vartheta \psi 470 \mathrm{deg}, \vartheta \psi 480 \mathrm{deg}$, and $\vartheta \psi 490 \mathrm{deg}$ two distinct frequencies $f_{n}$ and $f_{Q}$ are observed. This can be explained by the unique crack behavior due to a different exciting torsional 

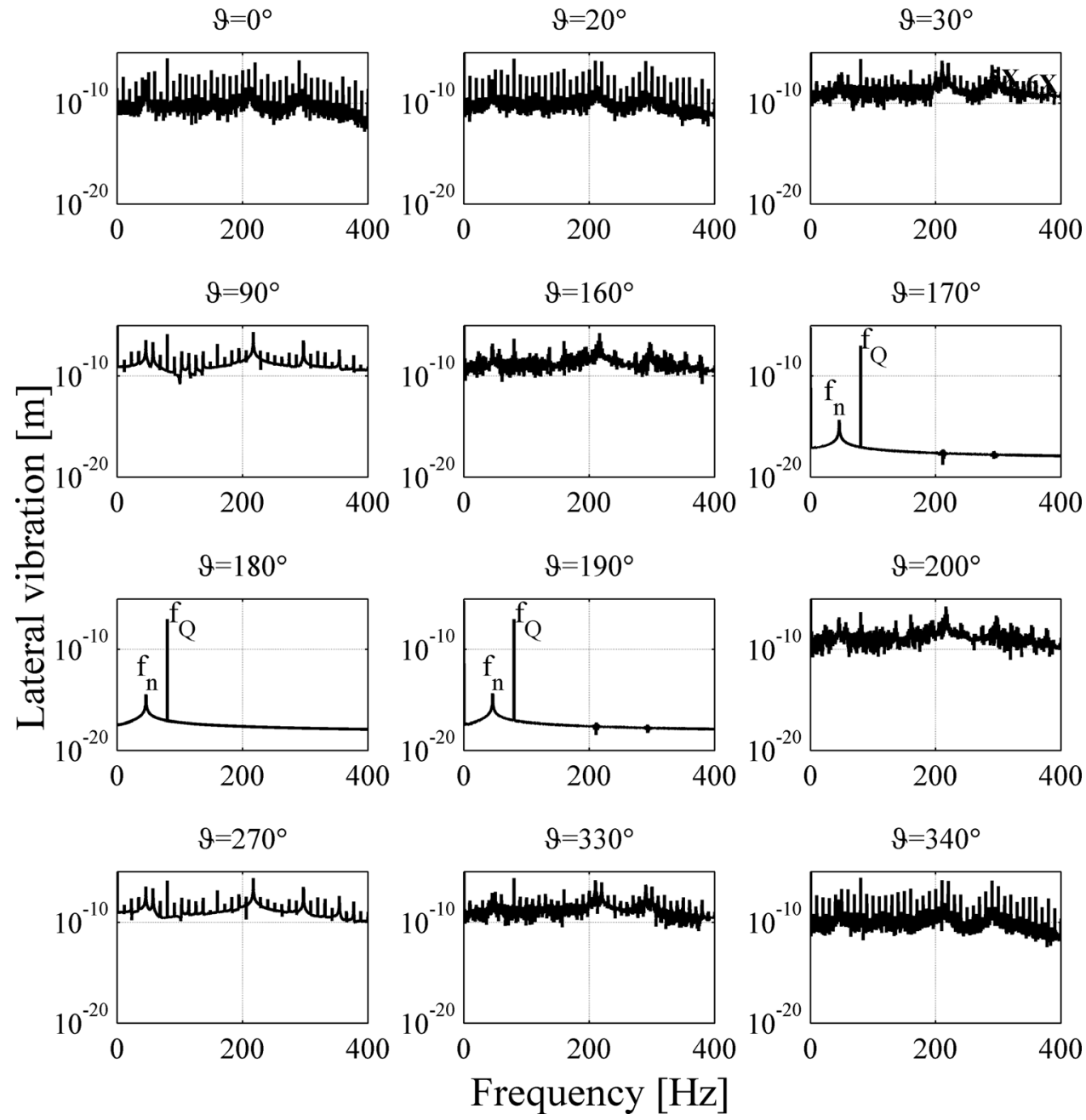

Fig. 13 Bending response for different angles $\vartheta ; 40 \%$ crack; $f_{Q}=80 \mathrm{~Hz}$

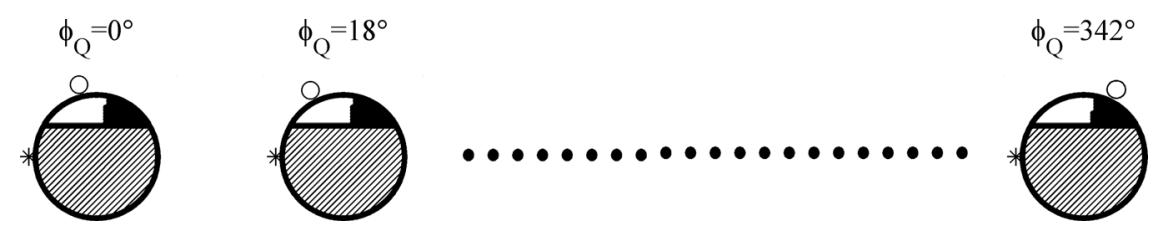

Fig. 14 Crack breathing at subsequent phases of the exciting torque; $25 \%$ crack; $f_{Q}=60 \mathrm{~Hz} ; \vartheta=90 \mathrm{deg}$

frequency. To illustrate this behavior, the crack opening/closing at subsequent phases $\phi_{Q}$ of the exciting torque has been calculated, and is presented in Figs. 14-17. For the 25\% cracked shaft excited with a $60 \mathrm{~Hz}$ torsional frequency, the crack remains partially open during the whole period of the exciting torque. This is shown in Fig. 14 for the angular location $\vartheta \notin 90 \mathrm{deg}$, where the crack remains half open. For other angular locations the crack behavior is similar, i.e., it remains partially open, yet the extent of this opening changes for various $\vartheta$. The behaviors of the $25 \%$ cracked shaft excited with $80 \mathrm{~Hz}$ as well as the $40 \%$ cracked shaft excited with $60 \mathrm{~Hz}$ are similar, though not presented here.

However, for the $40 \%$ cracked shaft excited with an $80 \mathrm{~Hz}$ torsional frequency, the crack behavior changes radically. For most angular locations of the lateral force the crack randomly opens and closes during one period of the exciting torque. This is presented in Figs. 15 and 16 for $\vartheta \nLeftarrow 0$ deg and $\vartheta \nLeftarrow+90$ deg. Such behavior results in an almost white noise spectrum observed in a vibration response (Fig. 13 for $\vartheta$ from $0 \mathrm{deg}$ to $160 \mathrm{deg}$ and from $200 \mathrm{deg}$ to $340 \mathrm{deg}$ ). For angular locations between $\vartheta(\neq 470 \mathrm{deg}$ and $\vartheta \notin 490$ deg the crack remains constantly closed (Fig. 17), what results in simple vibration spectra (Fig. 13 for $\vartheta \psi$ from $170 \mathrm{deg}$ to $190 \mathrm{deg}$ ) consisting of two components $f_{n}$ and $f_{Q}$ only.

Hence, the frequency of the exciting torque and other parameters should be selected very carefully, which suggests further studies on the proposed method. Nevertheless, it should be emphasized that if the parameters are chosen correctly then the proposed method can be presumably applied to effectively diagnose the health of the machine. 


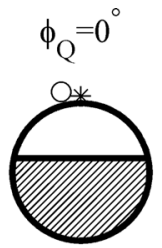

$\phi_{\mathrm{Q}}=90^{\circ}$

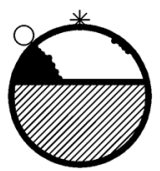

$\phi_{\mathrm{Q}}=180^{\circ}$

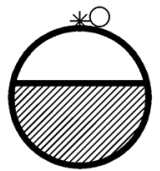

$\phi_{\mathrm{Q}}=270^{\circ}$

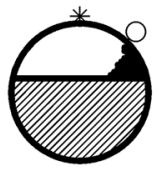

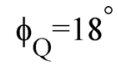

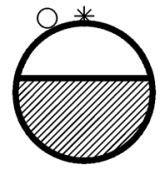

$\phi_{\mathrm{Q}}=108^{\circ}$

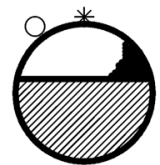

$\phi_{\mathrm{Q}}=198^{\circ}$

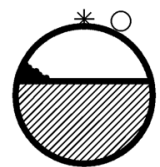

$\phi_{\mathrm{Q}}=288^{\circ}$

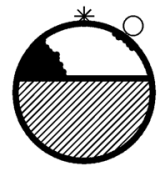

$\phi_{\mathrm{Q}}=36^{\circ}$

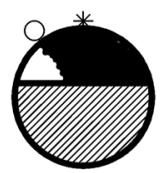

$\phi_{\mathrm{Q}}=126^{\circ}$

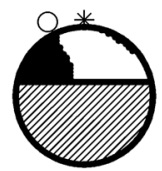

$\phi_{\mathrm{Q}}=216^{\circ}$

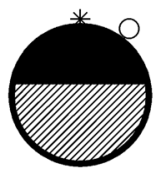

$\phi_{\mathrm{Q}}=306^{\circ}$

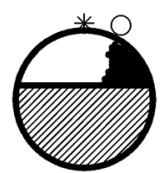

$\phi_{\mathrm{Q}}=54^{\circ}$

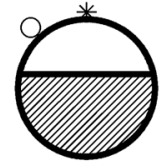

$\phi_{\mathrm{Q}}=144^{\circ}$

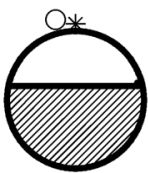

$\phi_{\mathrm{Q}}=234$

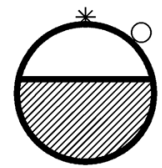

$\phi_{\mathrm{Q}}=324^{\circ}$

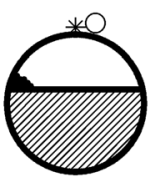

$\phi_{\mathrm{Q}}=72^{\circ}$

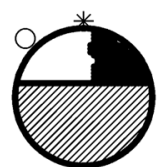

$\phi_{\mathrm{Q}}=162^{\circ}$

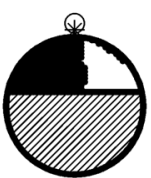

$\phi_{\mathrm{Q}}=252^{\circ}$

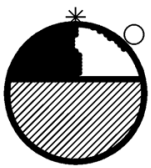

$\phi_{\mathrm{Q}}=342^{\circ}$

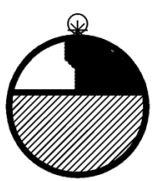

Fig. 15 Crack breathing at subsequent phases of the exciting torque; $40 \%$ crack; $f_{Q}=80 \mathrm{~Hz}$; $\vartheta=\theta \mathrm{deg}$

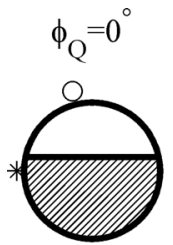

$\phi_{\mathrm{Q}}=90^{\circ}$

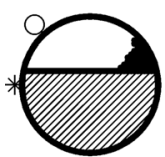

$\phi_{\mathrm{Q}}=180^{\circ}$

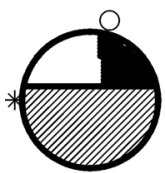

$\phi_{\mathrm{Q}}=270^{\circ}$
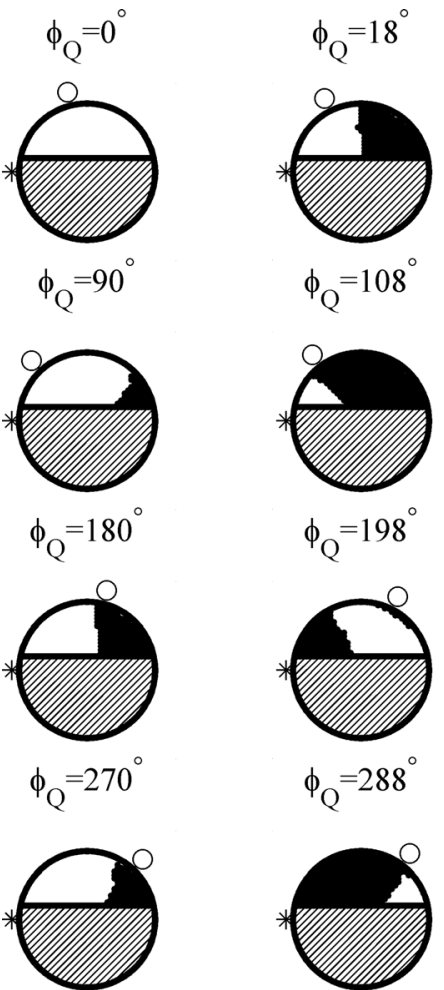

$\phi_{\mathrm{Q}}=108^{\circ}$

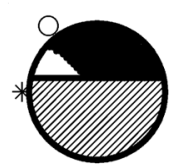

$\phi_{\mathrm{Q}}=198^{\circ}$

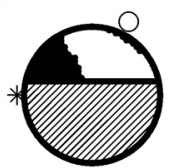

$\phi_{\mathrm{Q}}=288^{\circ}$

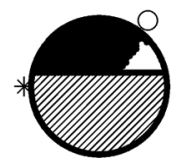

$\phi_{\mathrm{Q}}=54^{\circ}$

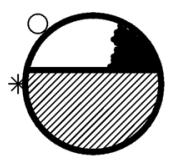

$\phi_{\mathrm{Q}}=144^{\circ}$

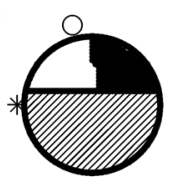

$\phi_{\mathrm{Q}}=234^{\circ}$

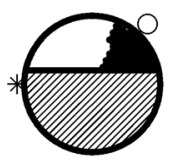

$\phi_{\mathrm{Q}}=324^{\circ}$

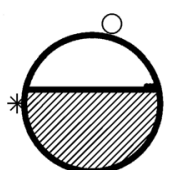

$\phi_{\mathrm{Q}}=72^{\circ}$

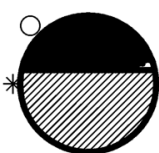

$\phi_{\mathrm{Q}}=162^{\circ}$

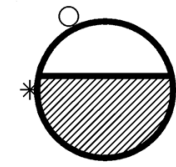

$\phi_{\mathrm{Q}}=216^{\circ}$

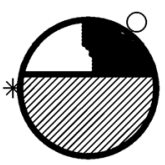

$\phi_{\mathrm{Q}}=306^{\circ}$
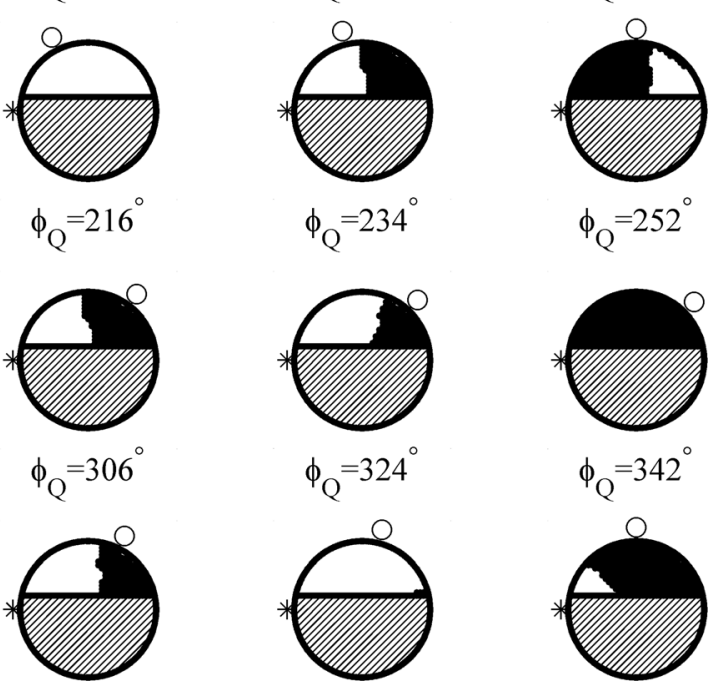

$\phi_{\mathrm{Q}}=342^{\circ}$

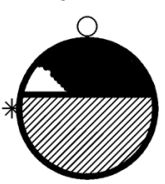

Fig. 16 Crack breathing at subsequent phases of the exciting torque; $40 \%$ crack; $f_{Q}=80 \mathrm{~Hz}$; $\vartheta=90 \mathrm{deg}$ 


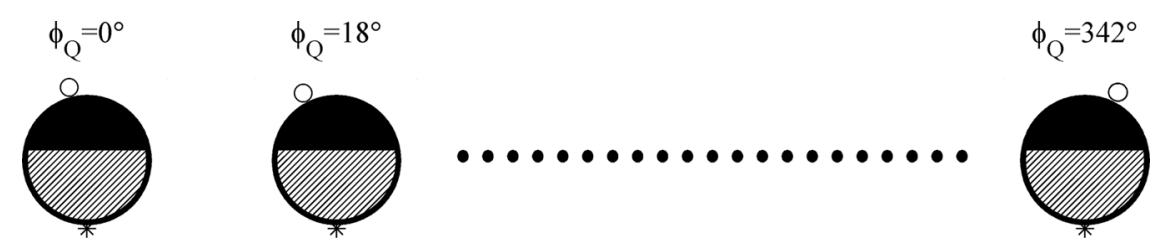

Fig. 17 Crack breathing at subsequent phases of the exciting torque; $40 \%$ crack; $f_{Q}=\mathbf{8 0 ~ H z} \boldsymbol{\vartheta}=\mathbf{1 8 0} \mathrm{deg}$

\section{Conclusions}

The main advantage of the proposed RFE approach is its simplicity. The model of the rotor is obtained quickly and intuitively using a uniform procedure. The crack is represented by a selected number of small spring-damping elements located between its lips. Introduction of several subsequent SDEs into the crack model is easy and is performed in a very systematic way that is natural for the RFE method. Modeling of the crack breathing mechanism using deformations of individual small SDEs is straightforward and results in fast computations involving only simple matrix operations. No complicated calculations of integrals for flexibilities, complex formulas for stress intensity factors, or so on, are required. Although fast and simple, the RFE method ensures very good accuracy as well. This has been preliminarily confirmed by the experimental and numerical results for the frequency transfer functions.

The presented approach amplifies the rotor sensitivity to the presence of crack and its propagation. Application of controlled deflection of the nonrotating shaft, excited by small amplitude harmonic torque, produces the unique patterns of coupled bending vibrations. If the deflection is applied in a direction corresponding to partial opening of the crack, the maximum amplification and the appearance of the multiples of the torsional frequency in the bending spectrum are observed. On the other hand, the minimum coupled bending amplitudes are observed if the deflection is directed in a way ensuring the fully opening or closing of the crack. Such behavior can be explained by the fact that in a case of a partially open crack, the multiples of the forced frequency appear quite naturally in the torsional spectrum. These frequencies are transformed by the off diagonal nonzero elements of the stiffness matrix to the coupled bending vibrations resulting in the same multiples in the bending vibration spectra. The coupling between the bending and torsional vibrations takes place only if the cracked shaft is considered, as only then the off diagonal nonzero elements appear in the stiffness matrix.

Since the technique requires simultaneous application of the harmonic torsional excitation and the constant magnitude load to the nonrotating rotor, it is not an online method and it can be used only during a major machine overhaul. The lateral force deflecting the shaft should be applied by a noncontacting force actuator, as for example an active magnetic bearing actuator. By proper controlling of the AMB, the angular direction of the applied force can be conveniently changed. The application of harmonic torque requires dedicated torsional noncontact exciters, similar to those developed by Sihler et al. [26] or Gaddis et al. [27], where the electromagnetic or piezoelectric actuators have been applied.

Numerical results confirm the potential of the proposed approach. The changes in coupled bending vibrations are observed only for the cracked shaft. However, further analysis is needed to determine the most optimal amplitude of the external force inducing the shaft deflection, the amplitude and frequency of the exciting torque, the axial location of the application for these forces, the placement of the measuring probes, etc.

The experimental validation of the proposed method is underway, as well as its extension to the case of rotating shafts. The approach has potential to become a robust technique for the continuous structural health monitoring of the rotors, without putting the machine out of service. The authors believe that the applica- tion of the method would allow one not only to detect the presence of the crack but also to identify its angular location.

\section{Nomenclature}

$A_{Q}=$ samplitude of the exciting torque

$a=$ erack depth

$\mathbf{D}=$ tamping matrix of the rotor

$d_{B}=$ radial damping coefficient of ball bearings

$d_{T}=$ torsional damping coefficient of the left ball bearing

$E=$ Young's modulus of the rotor's material

$F_{e x}=$ texternal lateral force deflecting the shaft

$\mathbf{F}_{e x}=$ vector of the external lateral force deflecting the shaft

$f_{n}=$ ffrst bending natural frequency of the rotor

$f_{Q}=$ frequency of the exciting torque

$f_{T}=$ first torsional natural frequency of the rotor

$\mathbf{G}=$ wector of gravity forces loading the rotor

$\mathbf{K}=$ stiffness matrix of the rotor

$k_{B}=$ radial stiffness coefficient of ball bearings

$k_{C, j}=$ stiffness coefficient of the big SDE in the $j$ th direction

$k_{k, j}=$ stiffness coefficient of the $k$ th SDE in the $j$ th direction

$k_{T}=$ torsional stiffness coefficient of the left ball bearing

$k_{U, j}=$ stiffness coefficient of the uncracked shaft in the $j$ th direction

$\mathbf{M}=$ mass matrix of the rotor

$n_{C}=$ tnumber of small SDEs modeling the crack

$n_{Q}=$ tmumber of cycles of the exciting torque

$Q_{T}=$ harmonic torque exciting the shaft

$\mathbf{Q}_{T}=$ wector of the harmonic torque exciting the shaft

$\mathbf{q}=$ wector of generalized displacements of centers of masses of RFEs

$\mathbf{q}_{p}=$ vector of displacements of the $p$ th RFE

$\mathbf{q}_{r}=$ wector of displacements of the $r$ th RFE

$R=$ shaft radius

$\mathrm{RFE}=$ rigid finite element

SDE $=$ spring-damping element

$\mathbf{T}_{r, k}=$ transformation matrix from $y_{k, 1}, y_{k, 2}, y_{k, 3}$ to $x_{r, 1}$, $x_{r, 2}, x_{r, 3}$ coordinates

$\mathbf{T}_{p, k}=$ transformation matrix from $y_{k, 1}, y_{k, 2}, y_{k, 3}$ to $x_{p, 1}$, $x_{p, 2}, x_{p, 3}$ coordinates

$t=$ time

$\Delta w_{k, 1}=$ translational deformation of the $k$ th SDE along axis $y_{k, 1}$

$\Delta \mathbf{w}_{k}=$ wector of deformations of the $k$ th SDE

$x_{r, 1}, x_{r, 2}, x_{r, 3}=$ axes of the local coordinate system of the $r$ th RFE

$y_{k, 1}, y_{k, 2}, y_{k, 3}=$ axes of the local coordinate system of the $k$ th SDE

$\alpha_{D}, \beta_{D}=$ «oefficients of the damping matrix $\mathbf{D}$

$\vartheta=$ kangle of the lateral force application

$\mu=$ relative depth of the crack

$\nu \notin=$ Poisson's ratio of the rotor's material

$\rho=$ tensity of the rotor's material

$\phi_{Q}=$ phase of the exciting torque 


\section{References}

[1] Bently, D. E., and Muszynska, A., 1986, "Detection of Rotor Cracks," Proceedings of Texas A\&M University 15th Turbomachinery Symposium and Short Courses, Corpus Christi, TX, pp. 129-139.

[2] Saavedra, P. N., and Cuitino, L. A., 2002, "Vibration Analysis of Rotor for Crack Identification,” J. Vib. Control, 8(1), pp. 51-67.

[3] Bachschmid, N., Pennacchi, P., and Tanzi, E., 2010, Cracked Rotors: A Survey on Static and Dynamic Behaviour Including Modelling and Diagnosing, Springer, Berlin

[4] Bachschmid, N., Pennacchi, P., Tanzi, E., and Vania, A., 2000, "Identification of Transverse Crack Position and Depth in Rotor Systems," Meccanica, 35, pp. $563-582$

[5] Söffker, D., Bajkowski, J., and Müller, P. C., 1993, "Detection of Cracks in Turborotors-A New Observer-Based Method," ASME J. Dyn. Syst. Measure. Control, 3, pp. 518-524.

[6] Kulesza, Z., and Sawicki, J. T., 2010, "Auxiliary State Variables for Rotor Crack Detection," J. Vib. Control, 17(6), pp. 857-872.

[7] Loparo, K. A., Adams, M. L., Lin, W., Abdel-Magied, M. F., and Afshari, N., 2000, "Fault Detection and Diagnosis of Rotating Machinery," IEEE Trans. Ind. Electron., 47(5), pp. 1005-1014.

[8] He, Y., Guo, D., and Chu, F., 2001, "Using Genetic Algorithms to Detect and Configure Shaft Crack for Rotor-Bearing System," Comput. Methods Appl. Mech. Eng., 190, pp. 5895-5906.

[9] Litak, G., and Sawicki, J. T., 2009 "Intermittent Behaviour of a Cracked Rotor in the Resonance Region," Chaos Solitions Fractals, 42, pp. 1495-1501.

[10] Xiang, J., Zhong, Y., Chen, X., and He, Z., 2008, "Crack Detection in a Shaft by Combination of Wavelet-Based Elements and Genetic Algorithm," Int. J. Solids Struct., 45, pp. 4782-4795.

[11] Guo, D., and Peng, Z. K., 2007, "Vibration Analysis of a Cracked Rotor Using Hilbert-Huang Transform," Mech. Syst. Signal Process., 21, pp. 3030-3041.

[12] Plaut, R. H., 1995, "Parametric, External and Combination Resonances in Coupled Flexural and Torsional Oscillations of an Unbalanced Rotating Shaft," J. Sound Vib., 183(5), pp. 889-897.

[13] Ishida, Y., and Inoue, T., 2006, "Detection of a Rotor Crack Using a Harmonic Excitation and Nonlinear Vibration Analysis," ASME J. Vib. Acoust., 128, pp. 741-749.
[14] Sawicki, J. T., Friswell, M. I., Kulesza, Z., Wroblewski, A., and Lekki, J. D., 2011, "Detecting Cracked Rotors Using Auxiliary Harmonic Excitation," J. Sound Vib., 330, pp. 1365-1381.

[15] Sawicki, J. T., Storozhev, D. L., and Lekki, J. D., 2011, "Exploration of NDE Properties of AMB Supported Rotors for Structural Damage Detection," ASME J. Eng. Gas Turbines Power, 133(10), p. 102501.

[16] Sawicki, J. T., and Baaklini, G. Y., 2004, "Coupled Lateral and Torsiona Vibrations of a Cracked Rotor," Proceedings of ASME Turbo Expo 2004, Power for Land, Sea, and Air. June 14-17, Vienna, Austria.

[17] Darpe, K., Gupta, K., and Chawla, A., 2004, "Coupled Bending, Longitudina and Torsional Vibrations of a Cracked Rotor," J. Sound Vib., 269, pp. 33-60.

[18] Kruszewski, J., Sawiak, S., and Wittbrodt, E., 1999, "The Rigid Finite Element Method in Dynamics of Structures," WNT, Warsaw, Poland (in Polish).

[19] Wittbrodt, E., Adamiec-Wójcik, I., and Wojciech, S., 2006, Dynamics of Flexible Multibody Systems: Rigid Finite Element Method, Springer, Berlin.

[20] Grabowski, B., 1984 “The Vibrational Behaviour of a Rotating Shaft Containing a Transverse Crack," Dynamics of Rotors-Stability System Identification (CISM Courses and Lectures, Vol. 273), O. Mahrenholtz, ed., Springer, New York.

[21] Gasch, R. A., 1993, "A Survey of the Dynamic Behaviour of a Simple Rotating Shaft With a Transverse Crack," J. Sound Vib., 160(2), pp. 313-332.

[22] Mayes, I. W., and Davies, W. G. R., 1984, "Analysis of the Response of a Multi-Rotor-Bearing System Containing a Transverse Crack in a Rotor,” J. Vib. Acoust. Stress Reliability Design, 83 DET 84, pp. 139-145.

[23] Nelson, H. D., and McVaugh, J. M., 1976, "The Dynamics of Rotor Bearing Systems Using Finite Elements," ASME J. Eng. Ind., 98, pp. 593-600.

[24] Wu, X., Sawicki, J. T., Friswell, M. I., and Baaklini, G. Y., 2005, "Finite Element Analysis of Coupled Lateral and Torsional Vibrations of a Rotor With Multiple Cracks," Proceedings of GT2005 ASME Turbo Expo 2005: Power for Land, Sea and Air, June 6-9, Reno, NV. Paper No. GT2005-68839, pp. 841-850.

[25] Dimarogonas, D., and Paipetis, S. A., 1983, Analytical Methods in Rotor Dynamics, Applied Science Publishers, London.

[26] Sihler, C., 2005, "A Novel Torsional Exciter for Modal Vibration Testing of Large Rotating Machinery," Mech. Syst. Signal Process., 20, pp. 1725-1740.

[27] Gaddis, T. W., Nelson, K. I., and Thomas, G. W., 1996, "Vibration Testing on Rotating Machine Components," US Patent 5,553,501, United Technologies Corporation. 